\title{
Assertive community treatment for high users of inpatient services had different outcomes at different types of hospitals
}

Rosenheck RA, Neale MS. Cost-effectiveness of intensive psychiatric community care for high users of inpatient services. Arch $G$ Psychiatry 1998 May;55:459-66.

\section{Question}

What are the effects of intensive psychiatric community care (IPCC) - a programme resembling assertive community treatment (ACT) - on clinical and social functioning, and costs for high users of psychiatric inpatient care?

\section{Design}

Randomised controlled trial with 2 years follow up.

\section{Setting}

6 general Veterans Affairs (VA) hospitals (offering acute psychiatric care) and 4 neuropsychiatric VA hospitals (offering longer term care).

\section{Patients}

873 patients (mean age 49 y) who were receiving inpatient care and had diagnoses other than substance abuse or brain disease. All patients were high users of inpatient services. Patients from neuropsychiatric hospitals $(n=345)$ were older, had poorer functioning, and were heavier users of inpatient care than those from general hospitals $(n=528)$.

\section{Intervention}

454 patients were allocated to IPCC and 419 to standard care. Monitoring of programme implementation showed that the IPCC programmes at all but 2 sites closely resembled ACT model programmes. IPCC teams consisted of social workers, nurses, and part time psychiatrists. Patients in the control group continued with standard inpatient treatment and follow up.

\section{Main cost and outcome measures}

Clinical and social outcomes were assessed by interview every 6 months. National computerised data were used to assess health service use, healthcare costs, and non-health costs. Cost data were analysed from the perspectives of the healthcare system and society. Costs are in US dollars.

\begin{abstract}
Main results
An analysis of data from all time points showed no difference in clinical or social outcome at either general or neuropsychiatric sites (except for 1 difference favouring IPCC at general sites on a non-standard social functioning scale). An analysis based on endpoint data alone, however, showed that at general sites IPCC was superior for symptoms $(\mathrm{p}<0.001)$, social functioning $(\mathrm{p}<0.001)$, and satisfaction with care $(\mathrm{p}<0.02)$. The IPCC group used less hospital care at neuropsychiatric sites (320 v 513 days, $\mathrm{p}<0.001)$ but not at general sites. Total societal costs were lower for the IPCC group at neuropsychiatric sites $(\$ 82454 v$ $\$ 116651, \mathrm{p}<0.001)$ but greater at general sites $(\$ 51537 v$ $\$ 46491, \mathrm{p}<0.01)$. However, IPCC costs at general sites became $\$ 38$ lower than standard care when 2 sites that had failed to implement IPCC were excluded.
\end{abstract}

\section{Conclusions}

At neuropsychiatric sites where patients were older and had lower functioning, intensive psychiatric community care (IPCC) (an intervention that resembled assertive community treatment) resulted in cost savings but no clinical or social improvements. At general sites (acute care hospitals) IPCC led to some clinical and social improvement, but did not reduce costs.

Source of funding: no external funding.

For correspondence: Dr R Rosenheck, Northeast Program Evaluation Center, 950 Campbell Avenue, West Haven, CT 06516, USA. Fax +1 2039373433.

\section{Commentary}

Rosenheck and Neale report the outcomes of 2 multicentre trials in high users of acute psychiatric care and users of long stay hospital care. The intervention is called IPCC but is more precisely described as assertive community treatment (ACT). ACT differs from most other case management approaches because it is a precisely defined model of care-fidelity to which can be measured. ${ }^{1}$ The key features of ACT are multidisciplinary teams, case sharing, assertive outreach, low caseloads, and an emphasis on rehabilitation in vivo. Although ACT has been extensively evaluated, these 2 trials are notable for their size, long follow up, few dropouts, and efforts to measure the fidelity of programme implementation. The implications of the trials will be considered in turn.

(i) ACT for high users of acute psychiatric care. A recent meta-analysis of relevant trials shows that ACT increases satisfaction and social functioning in this population while also reducing hospital use and costs. ${ }^{2}$ The present trial confirms the findings on satisfaction and social functioning while, for the first time, showing that long term ACT also improves psychiatric symptoms. The trial does not confirm a reduction in inpatient care, but the cost data suggest that this may be because of incomplete implementation at 2 of 6 sites. Overall, the trial upholds ACT as clinically effective and, at worst, cost neutral.

(ii) ACT for higher users of long term care. The same meta-analysis shows that although ACT reduced inpatient care in this population, insufficient evidence exists to support cost effectiveness or clinical effectiveness. ${ }^{2}$ Rosenheck and Neale's findings confirm the reduction in inpatient care and show a large cost reduction. The trial is large enough to suggest that no major clinical benefits or harms exist. The trial therefore shows ACT to be less costly and equally effective as long term inpatient psychiatric care.

This study raises 2 further points of note. The first is that the evidence supports intensive case management only when it is faithful to the ACT model. ${ }^{3}$ Secondly, ACT is an expensive intervention that pays for itself by reducing the use of another expensive intervention (inpatient care). ${ }^{2}$ The cost advantage of ACT therefore depends on the amount of hospital care used by the target population so that it may become a very expensive treatment when applied to low users of inpatient care.

Max Marshall, MD University of Manchester Lancashire, UK

1 McGrew JH, Bond GR, Dietzen L, et al.J Consult Clin Psychol 1994;62:670-8.

2 Marshall M, Lockwood A. In: Cochrane Library, Marshal M, Lockwood A. In: Cochrane Libre
issue 2, 1998. Oxford: Update Software.

3 Marshall M, Gray A, Lockwood A, et al. In: Cochrane Library, issue 2, 1998. Oxford: Update Software. 